\title{
Front-view Gait Recognition
}

\author{
Michela Goffredo, John N. Carter and Mark S. Nixon
}

\begin{abstract}
We present a new method for front-view gait biometrics which uses a single non-calibrated camera and extracts unique signatures from descriptors of a silhouette's deformation. The proposed approach is particularly suitable for identification by gait in the real world, where the advantages of completely unobtrusiveness, remoteness and covertness of the biometric system preclude the availability of camera information and where the CCTV images usually present subjects from an upper front-view. Tests on three different gait databases with subjects walking towards the camera have been performed. The obtained results, with mean CCR of $96.3 \%$, show that gait recognition of individuals observed the front can be achieved without any knowledge of camera parameters. Moreover, the method has been applied to three different walking directions and the results have been compared with the algorithms found in literature. The performance of the proposed system is particularly encouraging for its appliance in surveillance scenarios.
\end{abstract}

\section{INTRODUCTION}

Gait biometrics, which concerns recognizing individuals by the way they walk is a particularly challenging research area, where the potential for personal identification is supported by a rich literature, including medical and psychological studies [11], [26]. The completely unobtrusiveness without any subject cooperation or contact for data acquisition make gait particularly attractive for identification purposes. Gait recognition techniques at the state of the art can be divided into 3D [5], [25] and 2D approaches [27], [43]. In the first group, identification relies on parameters extracted from the 3D limb movement. These methods use a large number of digital cameras and the 3D reconstruction is achieved after a camera calibration process. On the other hand, the 2D gait biometric approaches extract explicit features describing gait by means of human body models [6], [28] or silhouette shape [37], [39]. A rich variety of data has been collected for evaluation of $2 \mathrm{D}$ gait biometrics. The widely used and compared databases on gait recognition include: the University of South Florida [31]; Carnegie Mellon University [13]; and the University of Southampton [33] data. The majority of methods and databases found in the literature use a single camera positioned with specific orientations with respect to the subject's walking direction (generally capturing the walk from the lateral view) and a large number of papers for gait recognition have been published [16], [27].

In real surveillance scenarios, however, we need a system that operates in an unconstrained environment where maybe

M. Goffredo is with School of Electronics and Computer Science, University of Southampton, United Kingdom mg2 decs. soton. ac.uk

J. N. Carter is with School of Electronics and Computer Science, University of Southampton, United Kingdom jnc@ecs.soton.ac.uk

M. S. Nixon is with School of Electronics and Computer Science, University of Southampton, United Kingdom msndecs.soton.ac.uk there is no information regarding the camera [42] and where the subject walks freely. Since CCTV cameras are usually placed on the top corners of buildings or on corridors' ceiling [9], the subject's pose is generally captured from an upper front-view. Moreover, the recent applications of gait recognition in criminal investigation, like the case of the murderer of Swedish Foreign Minister Anna Lindh [23], the bank robber in Noerager (Denmark) [20] and the burglar in Lancashire (United Kingdom) [1], have shown how the frontview can be effectively used in forensic studies. Figure 1 shows some example images from CCTV which have been successfully used for criminal identification. Therefore, an automatic gait recognition system which does not depend on camera calibration and is able to recognise individuals walking towards the camera is attractive. $2 \mathrm{D}$ view-independent gait identification is a relatively new research area and the approaches found in literature tend to synthesise the sagittal view of the human body from other arbitrary views [12], [18], [35] or to extract gait parameters which are independent from the human pose [4], [38]. The first choice is justified by the fact that the lateral view has demonstrated the recognition capability in many works [27], [43]. However, it has been shown that a walking direction of $70^{\circ}$ (considering $0^{\circ}$ the lateral view) is the upper bound of these methods, thus the kinematic information from the front-view is not sufficient for the lateral reconstruction. There is a large number of silhouette-based approaches for 2D gait recognition [3], [21], [30], but only few are tested on front-view images [7], [34], [38]. They present interesting results on a very limitative number of subjects $(n=4)$ or recognition rates lower than $82 \%$ if tested on a relatively larger dataset $(n=20)$.

The aim of this paper is to propose a novel biometric system for the front-view gait recognition and test it on a large number of subjects walking towards a camera from different gait databases. The method is based on the ability of human beings to recognize others from the front-view, where the body shape and its temporal variations are basic contributors to recognition [11], [36]. The silhouette's variation is described via 3D moment invariants on the gait volume obtained as the set of temporal images. The gait signature, containing both static and dynamic parameters, is unique for every subject and includes information regarding both the upper and the lower limbs kinematics.

Comparative tests with other algorithms found in literature will show that the proposed method, even with a basic description of the silhouette's deformation, allows to recognise individuals by the way they walk not only from the front but also from others points of view. 


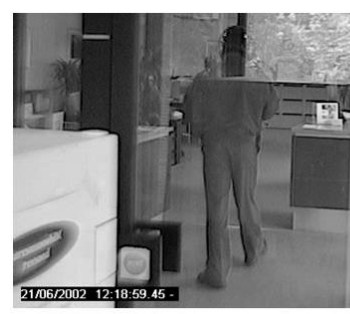

(a)

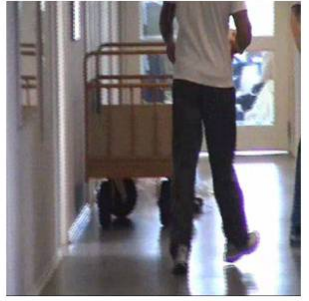

(b)

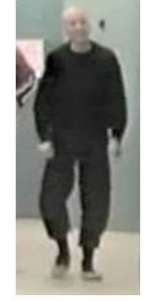

(c)

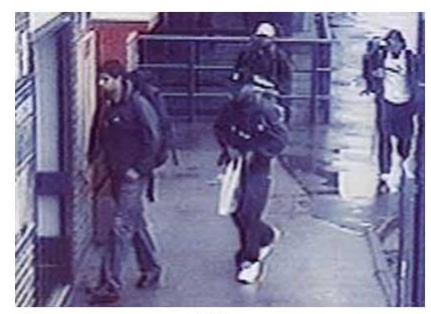

(d)

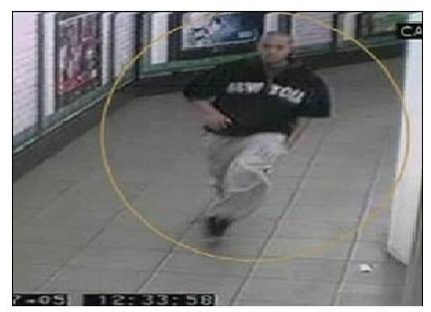

(e)

Fig. 1. Some CCVT's images successfully used for criminal identification. (a) and (b): the bank robber in Noerager during the robbery and later in the police station [20]; (c): the burglar in Lancashire [1]; (d) and (e): '7/7' terrorist bombings in London [1].

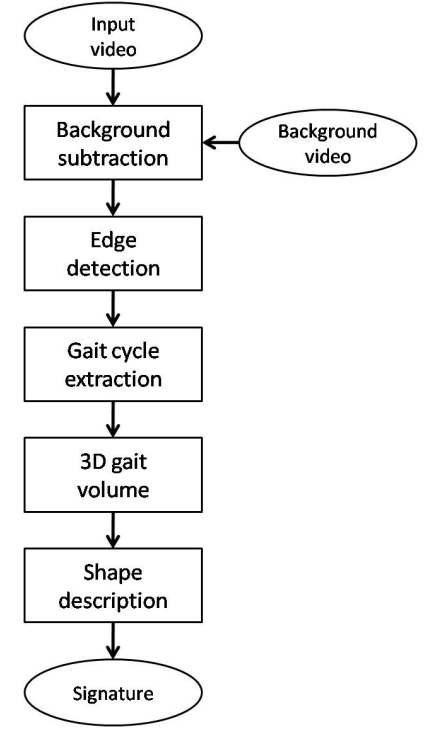

Fig. 2. Flow chart of the proposed method

\section{THE NEW METHOD}

The proposed method for the front-view gait analysis is based on two consecutive steps: the gait cycle detection and the gait volume description. Figure 2 shows the basic steps of the algorithm.

\section{A. Gait Cycle Detection}

The detection of the gait cycle is generally the first step in gait analysis given the periodic nature of the gesture. In order to extract the walking figure from the background image, a background subtraction method is necessary which can be generated by a variety of methods. We use a standard background subtraction technique to segment the body's silhouette from the video sequence since the dataset is captured in a controlled environment [2], [37]. More sophisticated approaches can be used in a more complex and less controlled environment.

Let $\mathbf{I}_{R G B}(\mathbf{x}, \mathbf{y}, \mathbf{T})$ be the RGB frames (of size $R \times C$ pixels) at time $\mathbf{T}=\left[t_{1}, t_{2}, \ldots, t_{\varphi}, \ldots, t_{F}\right]$ where $\left(x_{1}, y_{1}\right)$ is the topleft corner of the image. By applying a background subtraction method based on the threshold of the 3 components of the color space YUV [40], the binary image $\overline{\mathbf{S}}\left(\mathbf{x}, \mathbf{y}, t_{\varphi}\right)$

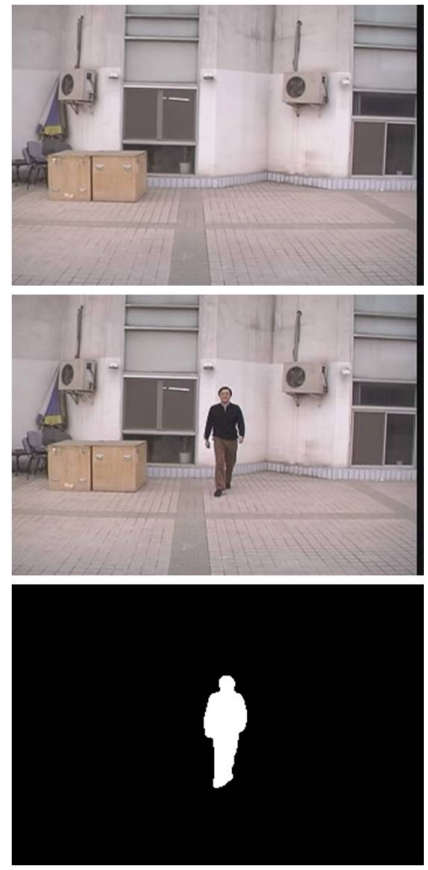

(a)

(b)

(c)

Fig. 3. An example of silhouette extraction: (a) Background image; (b) Original Image; (c) Extracted silhouette

has been extracted. The pixels $\left(\mathbf{x}_{s i l}, \mathbf{y}_{\text {sil }}\right)$ containing the human silhouette have been extracted as the object with maximum area [14]. Figure 3 shows an example background subtraction.

Medical studies [26], [29] suggests that human gait is a form of periodic motion and cyclic phases of the walking sequence can be extracted. Therefore, the body's pose during walking changes periodically and the upper and lower limbs move symmetrically [10], [17]. Since the width and height of the bounding box directly depend on the limb's fluctuation, we consider the gait fluctuation as a periodic function which depends on the silhouette width and height over time:

$$
\mathbf{C F}\left(t_{\varphi}\right)=\bar{H}\left(t_{\varphi}\right) \cdot \bar{W}\left(t_{\varphi}\right)
$$

where $\bar{H}\left(t_{\varphi}\right)$ and $\bar{W}\left(t_{\varphi}\right)$ are the silhouette's height and width at frame $t_{\varphi}$ after detrending and normalizating them to compensate the scale variation. The gait cycle is then the time between 2 successive minima of the $\mathbf{C F}$ function. Figure 4 

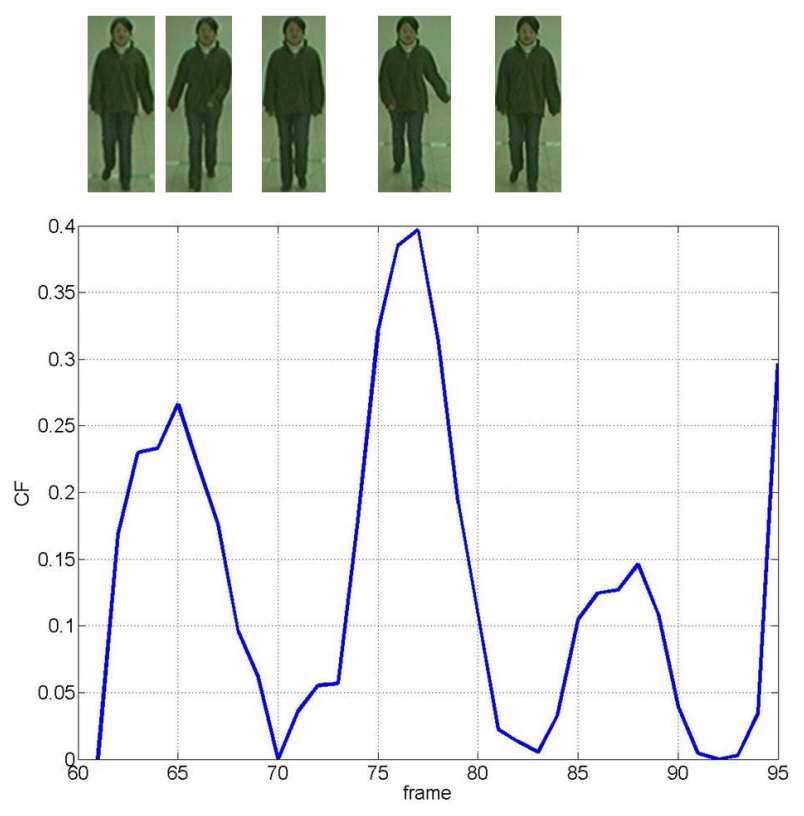

Fig. 4. Gait cycle detection via the periodic function $\mathbf{C F}$

shows CF versus time and the corresponding frames. The variability of $\mathbf{C F}$ is high because of the asymmetric limbs swing. Since the the positions of the minima correspond to the gait phase where the horizontal limbs' swing from the front-view can be considered null, the gait cycle is extracted successfully. Once a gait cycle is measured, a bounding box is placed around the silhouettes over one gait period and the cropped images are normalised with respect to the minimum height and processed by the gait volume descriptor.

\section{B. Gait Volume Description: signature extraction}

After having extracted the $G$ silhouettes belonging to one gait cycle $(g=1, \ldots, G)$, the $3 \mathrm{D}$ gait volume $\mathbf{V}(\mathbf{x}, \mathbf{y}, \mathbf{t})$ has been built by placing the edges points in a $3 \mathrm{D}$ space $(\mathbf{x}, \mathbf{y}, \mathbf{t})$ where $\mathbf{t}$ is defined as the percentage of gait cycle

$\mathbf{t}=\left[0,1 \cdot \frac{100}{G-1}, 2 \cdot \frac{100}{G-1}, \ldots,(g-1) \cdot \frac{100}{G-1}, \ldots, 100\right]$

and $\mathbf{x}$ and $\mathbf{y}$ are the horizontal and vertical axes normalized by the same factor so that $\mathbf{y}=[0, \ldots, 1]$ and the silhouette's proportions are maintained. The silhouettes' alignment is obtained by stacking the normalised bounding boxes over time. Therefore, each gait sequence corresponds to a spatiotemporal volume, whose description and interpretation is achieved via moment invariants.

The idea of using moments in shape recognition gained prominence when $\mathrm{Hu}$ [15] derived a set of invariants using the algebraic invariants. The property that they are independent of orientation has attracted much interest and then been widely used in various applications. In 1980, Sadjadi and Hall [32] first extended moment invariants from $2 \mathrm{D}$ to $3 \mathrm{D}$ and so far the 3D implementation has been used in brain morphometry [24] and complex shapes classification [19], [22], [41].

Three-dimensional moments of a sampled volume $\mathbf{x} \times \mathbf{y} \times \mathbf{t}$ that have a function $\mathbf{V}(\mathbf{x}, \mathbf{y}, \mathbf{t})$ is given as,

$$
m_{p q r}=\sum_{x \in \mathbf{x}} \sum_{y \in \mathbf{y}} \sum_{t \in \mathbf{t}} x^{p} \cdot y^{q} \cdot t^{r} \cdot \mathbf{V}(x, y, t)
$$

where $O=p \cdot q \cdot r$ is the moments' order.

The moments of $\mathbf{V}(\mathbf{x}, \mathbf{y}, \mathbf{t})$ translated by an amount $(a, b, c)$ are defined as

$$
\bar{m}_{p q r}=\sum_{x \in \mathbf{x}} \sum_{y \in \mathbf{y}} \sum_{t \in \mathbf{t}}(x+a)^{p} \cdot(y+b)^{q} \cdot(t+c)^{r} \cdot \mathbf{V}(x, y, t)
$$

so, the central moment $\mu_{p q r}$ can be computed from 4

$$
\mu_{p q r}=\sum_{x \in \mathbf{x}} \sum_{y \in \mathbf{y}} \sum_{t \in \mathbf{t}}(x-\bar{x})^{p} \cdot(y-\bar{y})^{q} \cdot(t-\bar{t})^{r} \cdot \mathbf{V}(x, y, t)
$$

substituting $a, b, c$ with the mean value of $\mathbf{x}, \mathbf{y}, \mathbf{t}$ respectively:

$$
\bar{x}=\frac{m_{100}}{m_{000}}, \bar{y}=\frac{m_{010}}{m_{000}}, \bar{t}=\frac{m_{001}}{m_{000}}
$$

Therefore, considering that the spatio-temporal volume $\mathbf{V}(\mathbf{x}, \mathbf{y}, \mathbf{t})$ is obtained from the silhouettes' edge, where the $j$ th point belonging to the edge of frame $t$ is $\left[x_{j}^{e d}(t), y_{j}^{e d}(t)\right]$ and $\mathbf{V}\left(x_{j}^{e d}(t), y_{j}^{e d}(t), t\right)=1$, the $3 \mathrm{D}$ central moments of the gait volume are

$$
\mu_{p q r}^{G A I T}=\sum_{t \in \mathbf{t}} \sum_{\mathbf{j}=1}^{E(t)}\left(x_{j}^{e d}(t)-\bar{x}\right)^{p} \cdot\left(y_{j}^{e d}(t)-\bar{y}\right)^{q} \cdot(t-\bar{t})^{r}
$$

where $E(t)$ is the number of edge's points at frame $t$.

Since $\mu_{p q r}^{G A I T}$ describes the front-gait independently from scale and translation, it is has been used as part of the gait signature. In order to distinguish the gait properties of each subject a feature vector consisting of $O+3$ features has been yielded. The signature is composed of the $3 \mathrm{D}$ central moments of the gait volume and some scale-dependent gait features, i.e. the number of frames for one gait cycle and the silhouette's height and width maximum increment.

Gait features have been classified via $k$-nearest neighbour classifier $(k-\mathrm{NN})$ where a test signature $\ell$ belongs to the class that minimizes the similarity distances between the test signature and all reference signatures. More sophisticated classifier could be employed, but the interest is to evaluate the discriminatory ability of the extracted features and to compare the results with methods found in literature. The leave-one-out cross validation rule has been used in order to obtain an unbiased estimation of recognition accuracy.

\section{EXPERIMENTAL TESTS}

\section{A. Gait Databases}

The performance of our algorithm has been tested using different gait databases available on line, where different camera's characteristics and light conditions are present:

- Small Gait Dataset of the University of Southampton [37]: 10 subjects, 10 videos per subject 
- CASIA-B Gait Database [2]: 50 subjects, 6 videos per subject

- CASIA-A Gait Database [2], also known as NLPR : 20 subjects, 4 videos per subject, 3 different camera views (front, lateral, $45^{\circ}$ )

The last dataset has been used in order to evaluate the performance of the algorithm with images captured from different view points, and to compare the results with other 2D methods found in literature. Example images from the databases are shown in figure 5.

\section{B. Results and Discussion}

The new method for front-view gait recognition has been applied to the video sequences, the gait cycle has been extracted, the gait volume built and the feature vector, including the moment invariants, has been calculated.

Since the extracted signature depends on the moment's order $O$, a quantitative study for choosing of the order which better describes the gait volume has been conducted. Figure 6 depicts the recognition rate by varying $O=$ $[0, \ldots,(10 \times 10 \times 10)+3]$ where $O$ includes the scaledependent gait features. The performance grows logarithmically and from $O=1000$ the CCR is $100 \%$. Therefore $p_{\max }=q_{\max }=r_{\max }=10$ have been chosen for the following experimental tests.

Figure 7 shows two gait volumes belonging to two different subjects of the CASIA-B database. The signatures are also depicted and confirm their considerable discriminating power for identifying individuals.

The percentage of recognition rate $(\% C C R)$ has been calculated on the 3 databases and the results are reported in table I, where $k$-NN classifiers with $k=1$ and $k=3$ have been considered. The whole results are very encouraging, with mean CCR equal to $96.3 \%$. The mean time for processing a signature is $2.05 \mathrm{~s} /$ frame (Matlab7 code processed with $2 \mathrm{GHz}$ Intel $\mathrm{CPU})$.

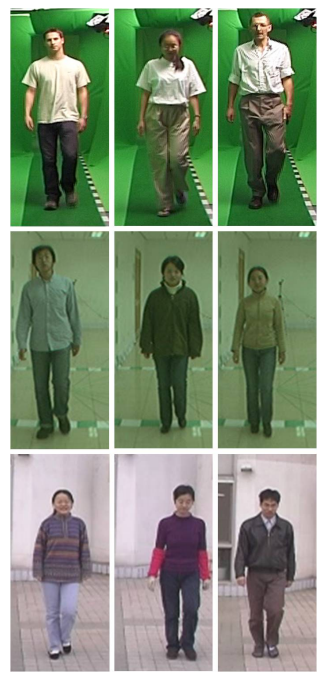

(a)

(b)

(c)

Fig. 5. Examples of front view images used in the experimental tests: (a) Small Southampton Database; (b) CASIA-B Database; (c) CASIA-A Database

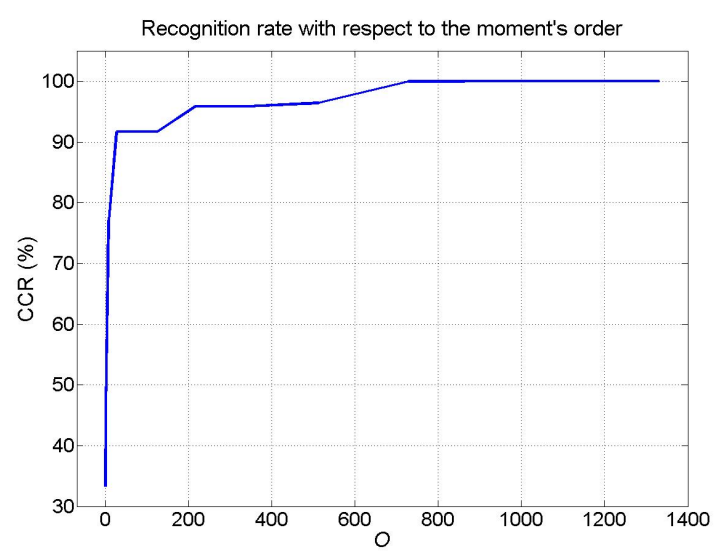

Fig. 6. \%CCR with respect to the moment's order (on Southampton database)
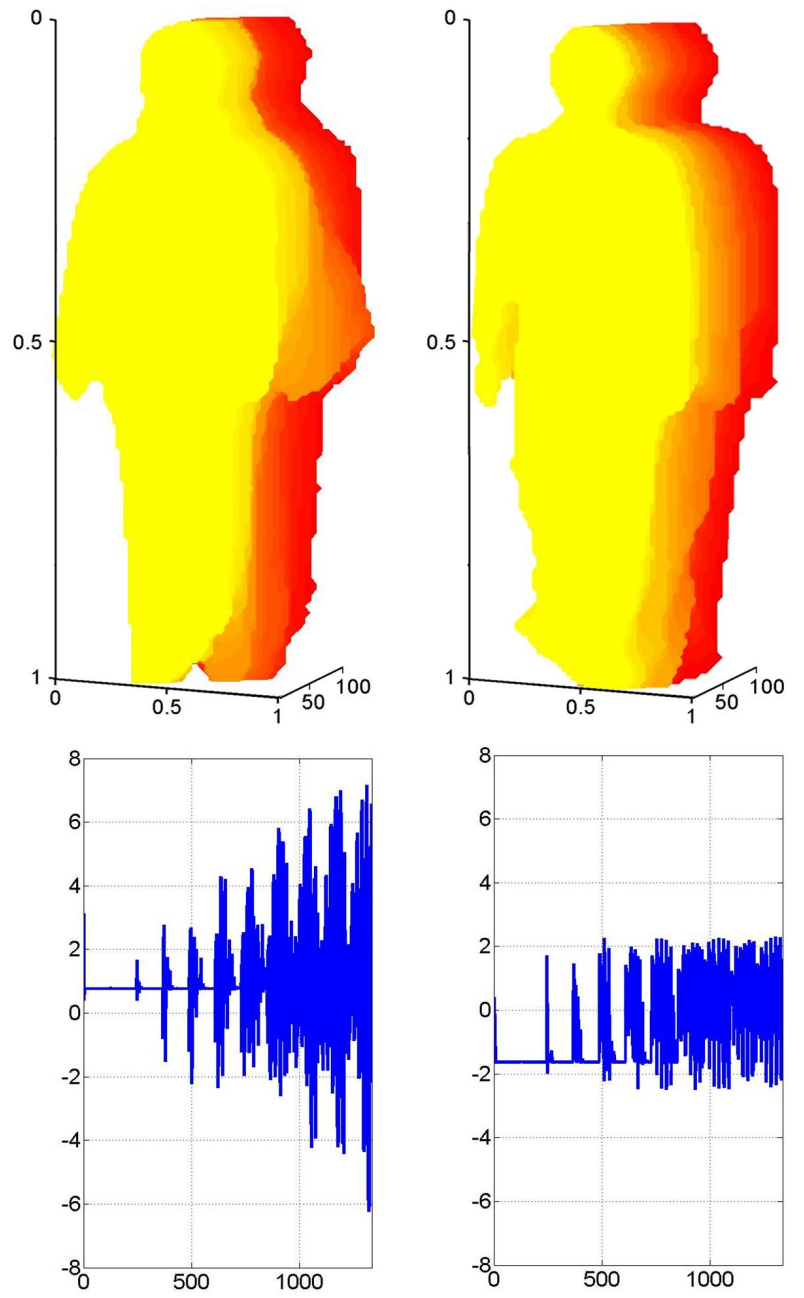

Fig. 7. Example of gait volumes and signatures for 2 subjects of CASIA-B database 


\begin{tabular}{|ccccc|}
\hline Database & Subjects & Videos & $\boldsymbol{k}=\mathbf{1}$ & $\boldsymbol{k}=\mathbf{3}$ \\
\hline Southampton & 10 & 10 & $100 \%$ & $100 \%$ \\
CASIA-A & 30 & 4 & $91.00 \%$ & $90.25 \%$ \\
CASIA-B & 50 & 6 & $97.92 \%$ & $89.58 \%$ \\
\hline
\end{tabular}

TABLE I

CCRS ON FRONT-VIEW IMAGES FROM DIFFERENT GAIT DATABASES

\begin{tabular}{|lccc|}
\hline Methods & Lateral & 45 deg & Front \\
\hline BenAbdelkader 2001 [3] & $72.50 \%$ & - & - \\
BenAbdelkader 2002 [4] & $82.50 \%$ & - & - \\
Collins 2002 [8] & $71.25 \%$ & - & - \\
Lee 2002 [21] & $87.50 \%$ & - & - \\
Phillips 2002 [30] & $78.75 \%$ & - & - \\
Wang 2003 [38] & $88.75 \%$ & $87.50 \%$ & $90.00 \%$ \\
Chen 2007 [7] & $92.50 \%$ & $95.00 \%$ & $65.00 \%$ \\
Our method & $100 \%$ & $97.50 \%$ & $91.00 \%$ \\
\hline
\end{tabular}

TABLE II

COMPARISON OF SEVERAL DIFFERENT ALGORITHMS ON THE CASIA-A DATABASE UNDER DIFFERENT VIEW ANGLES

To examine the performance of the proposed algorithm, comparative experiments have been evaluated. Seven recent methods for gait biometrics, that are silhouette-based and have used the same CASIA-A dataset (also known as NLPR), have been considered [3], [4], [7], [8], [21], [30], [38].

While the first five methods have been designed and tested exclusively for the lateral view, the latter two allow us to compare the performance of our approach with the front and the 45 degree views.

The results are summarized in table II. From table II, we can see that our method compares favourably with others. Obviously the performance decreases from the lateral to the front-view, because of the decrease of information regarding the leg swing, but the decrease of CCR is higher than the other methods. The performance of [38] is the only one having a different trend, where the front-view presents a better performance. To this extent, the performance trend of our method with respect to the camera view appears similar to [7], where the gait silhouettes are described by tiling one period gait subsequence in a $2 \mathrm{D}$ polar-plane.

The whole results demonstrate how the proposed approach allows the gait identification not only from the front-view, which is the main aim of this paper, but also from the lateral and 45 degrees views. Moreover, the recognition rates, with a mean value of $96.1 \%$, are above the ones found in literature and are particularly encouraging for future tests on larger databases.

\section{CONCLUSIONS AND FUTURE WORKS}

The remote and covert nature of gait as a biometric has continued the interest in this field. However, most approaches to date limit their application to the lateral view, or use camera calibration or even require the use of multiple cameras. Considering real scenarios, a system which estimates the gait parameters from a single view, without relying on the subject's pose or on camera calibration is required. Moreover, images from CCTV cameras usually present subjects from an upper front-view.

Therefore, this paper has introduced a novel method for the front-view gait biometrics where the subject's signature is extracted from the description of the silhouettes' deformation.

Tests on three different gait databases with subjects walking towards the camera have been performed and the obtained results have shown that gait recognition of individuals observed in the front-view can be achieved without any knowledge of camera parameters. Moreover, comparative results with algorithms found in literature reported that the approach can identify people also from the lateral and oblique view. Future developments will regard investigation of other techniques for the spatio-temporal volume description and further experimental tests in uncontrolled environments. The general applicability of the method and the obtained recognition rate are particularly encouraging for appliances in surveillance scenario.

\section{ACKNOWLEDGMENTS}

The study was supported by DTC through General Dynamics and uses the CASIA Gait Database collected by Institute of Automation, Chinese Academy of Sciences.

\section{REFERENCES}

[1] http://news.bbc.co.uk/.

[2] Casia gait database. online, 2006.

[3] C. BenAbdelkader, R. Cutler, H. Nanda, and L. S. Davis. Eigengait: Motion-based recognition of people using image self-similarity. In Proc Int Conf AVBPA, pages 284-294, 2001.

[4] C. BenAbdelkader, L. S. Davis, and R. Cutler. Motion-based recognition of people in eigengait space. In Proc IEEE Conf $F G$, pages 267-274, 2002.

[5] B. Bhanu and J. Han. Human recognition on combining kinematic and stationary features. In Proc Int Conf AVBPA, pages 600-608, 2003.

[6] I. Bouchrika and M. S. Nixon. Model-Based Feature Extraction for Gait Analysis and Recognition, volume 4418 of LNCS. Springer, 2007.

[7] S. Chen and Y. Gao. An invariant appearance model for gait recognition. Proc IEEE Conf Multimedia Expo, pages 1375-1378, 2007.

[8] R. Collins, R. Gross, and J. Shi. Silhouette-based human identification from body shape and gait. In Proc IEEE Conf FG, pages 351-356, 2002.

[9] M. Constant and P. Ridgeon. The Principles and Practice of CCTV. Paramount Publishing Ltd, 2000.

[10] D. Cunado, M. S. Nixon, and J. N. Carter. Automatic extraction and description of human gait models for recognition purposes. Comput. Vis. Image Underst., 90(1):1-41, 2003.

[11] J. Cutting and L. Kozlowski. Recognizing friends by their walk: gait perception without familiarity cues. Bull. Psychonom. Soc., 9:353356, 1977.

[12] M. Goffredo, N. Spencer, D. Pearce, J. N. Carter, and M. S. Nixon. Human Perambulation as a Self Calibrating Biometric., volume 4778 of LNCS. Springer, 2007.

[13] R. Gross and J. Shi. The cmu motion of body (mobo) database. Technical Report CMU-RI-TR-01-18, Robotics Institute, Carnegie Mellon University, Pittsburgh, PA, 2001.

[14] R. M. Haralick and L. G. Shapiro. Computer and Robot Vision, volume 1. Addison-Wesley, 1992.

[15] M.-K. Hu. Visual pattern recognition by moment invariants. IEEE Trans Information Theory, 8(2):179-187, 1962.

[16] W. Hu, T. Tan, L. Wang, and S. Maybank. A survey on visual surveillance of object motion and behaviors. IEEE Trans Systems Man Cybernetics, 34(3):334-352, 2004.

[17] K. M. Jackson, J. Joseph, and S. J. Wyard. The upper limbs during human walking. part 2: Function. Electromyogr Clin Neurophysiol., 23:435-46, 1983. 
[18] A. Kale, A. Chowdhury, and R. Chellappa. Towards a view invariant gait recognition algorithm. Proc IEEE Conf AVSS, pages 143-150, 2003.

[19] L. Keyes and A. Winstanley. Using moment invariants for classifying shapes on large-scale maps. Computers, Environment Urban Syst., 25:119-130, 2001.

[20] P. K. Larsen, E. B. Simonsen, and N. Lynnerup. Gait analysis in forensic medicine. volume 6491. SPIE, 2007.

[21] L. Lee and W. Grimson. Gait analysis for recognition and classification. Proc IEEE Conf FG, pages 148-155, 2002.

[22] Z. Li, Q. Zhang, Q. Hou, L. Diao, and H. Li. The shape analysis based on curve-structure moment invariants. Proc Int Conf ICNN\&B, 2:1139-1143, 2005.

[23] N. Lynnerup. Person identification by gait analysis \& photogrammetry. In Proc Conf Crime Solutions, 2006.

[24] J.-F. Mangin, F. Poupon, E. Duchesnay, D. Rivière, A. Cachia, D. L. Collins, A. C. Evans, and J. Régis. Brain morphometry using $3 \mathrm{~d}$ moment invariants. Medical Image Analysis, 8:187-196, 2004.

[25] L. Middleton, D. K. Wagg, A. I. Bazin, J. N. Carter, and M. S. Nixon. Developing a non-intrusive biometric environment. Proc IEEE Int Conf IROS, pages 723-728, 2006.

[26] M. P. Murray, A. B. Drought, and R. C. Kory. Walking patterns of normal men. J Bone Joint Surgery, 46:335, 1964.

[27] M. S. Nixon and J. N. Carter. Automatic recognition by gait. Proc. of the IEEE, 94(11):2013-2024, 2006.

[28] C. Orrite-Urunuela, J. del Rincon, J. Herrero-Jaraba, and G. Rogez. 2d silhouette and $3 \mathrm{~d}$ skeletal models for human detection and tracking. Proc IEEE Conf ICPR, 4:244-247, 2004.

[29] J. Perry. Gait Analysis: Normal and Pathological Function. McGraw Hill, New York, 1992.

[30] P. Phillips, S. Sarkar, I. Robledo, P. Grother, and K. Bowyer. Baseline results for the challenge problem of humanid using gait analysis. Proc IEEE Conf FG, pages 130-135, 2002.

[31] P. Phillips, S. Sarkar, I. Robledo, P. Grother, and K. Bowyer. The gait identification challenge problem: data sets and baseline algorithm. Proc Int Conf Pattern Recognition, 1:385-388 vol.1, 2002.

[32] F. A. Sadjadi and E. L. Hall. Three-dimensional moment invariants. IEEE Trans PAMI, 2:127-136, 1980.

[33] J. Shutler, M. Grant, M. S. Nixon, and J. N. Carter. On a large sequence-based human gait database. In Proc Int Conf Recent Advances in Soft Computing, pages 66-72, 2002.

[34] M. Soriano, A. Araullo, and C. Saloma. Curve spreads-a biometric from front-view gait video. Pattern Recognition Letters, 25(14):15951602, 2004.

[35] N. Spencer and J. Carter. Towards pose invariant gait reconstruction. Proc IEEE Conf ICIP, 3:III-261-4, 2005.

[36] S. V. Stevenage, M. S. Nixon, and K. Vince. Visual analysis of gait as a cue to identity. Applied Cognitive Psychology, 13(6):513-526, 1999.

[37] G. Veres, L. Gordon, J. Carter, and M. Nixon. What image information is important in silhouette-based gait recognition? Proc IEEE Conf CVPR, 2:II-776-II-782 Vol.2, 2004.

[38] L. Wang, T. Tan, W. Hu, and H. Ning. Automatic gait recognition based on statistical shape analysis. IEEE Trans IP, 12(9):1120-1131, 2003.

[39] L. Wang, T. Tan, H. Ning, and W. Hu. Silhouette analysis-based gait recognition for human identification. IEEE Trans PAMI, 25(12):15051518, 2003.

[40] C. Wren, A. Azarbayejani, T. Darrell, and A. Pentland. Pfinder: realtime tracking of the human body. Proc IEEE Conf FG, pages 51-56, 1996.

[41] D. Xu and H. Li. 3-d surface moment invariants. In ICPR (4), pages 173-176, 2006.

[42] Y. Yamada. Advanced method for improvement of obscure video image. Security Technology, 1999. Proceedings. IEEE 33rd Annual 1999 International Carnahan Conference on, pages 440-445, 1999.

[43] R. Zhang, C. Vogler, and D. Metaxas. Human gait recognition at sagittal plane. J Image Vision Comput, 25(3):321-330, 2007. 